\title{
Téoros
}

Revue de recherche en tourisme

\section{Formation et recherche en tourisme à l'UQAM : une ouverture sur le monde}

\section{Louis Jolin}

Volume 11, numéro 1, mars 1992

La formation en tourisme : à la croisée des chemins

URI : https://id.erudit.org/iderudit/1078942ar

DOI : https://doi.org/10.7202/1078942ar

Aller au sommaire du numéro

Éditeur(s)

Université du Québec à Montréal

ISSN

0712-8657 (imprimé)

1923-2705 (numérique)

Découvrir la revue

Citer cet article

Jolin, L. (1992). Formation et recherche en tourisme à l'UQAM : une ouverture sur le monde. Téoros, 11(1), 12-15. https://doi.org/10.7202/1078942ar d'utilisation que vous pouvez consulter en ligne.

https://apropos.erudit.org/fr/usagers/politique-dutilisation/ 


\section{Formation et recherche en tourisme à I'UQAM: une ouverture sur le monde}

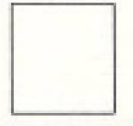

Les évolutions récentes dues au contexte de crise mondiale sont-elles exclusivement quantitatives ou, au contraire, s"accompagnent-elles, en s'en nourrissant, de mutations plus profondes, concernant les choix et les modes du tourisme? Les permanences, jusque-là très vigoureuses en matière de périodes de départ, de lieux de destination, de formules d'hébergement par exemple, vontelles s'estomper au profit d'une nouvelle dynamique touristique et d'un modelage différent de la carte mondiale? Les signes d'un tel bouleversementrestentaujourd' hui très modestes: le tourisme international a certes, beaucoup changé et continue à le faire, mais de manière toujours progressive, et souvent fort énigmatique, en fonction des logiques et des dynamiques sociales qui lui servent de cadre ${ }^{(1)}$.

C'est par ces mots que Georges Cazes termine son livre sur le tourisme international. Je les retiens pour débuter cet article sur les dimensions internationales de la formation en tourisme à l'UQAM. Oui, le tourisme international change et continuera de le faire ces prochaines années...mais bien malin celui qui pourra prévoir l'orientation précise. Dans les années soixante dix, des futurologues ${ }^{(2)}$ comme des experts de la Banque mondiale faisaient du tourisme un vecteur essentiel du développement mondial, une contribution vitale à la croissance du Tiers Monde..mais la crise internationale (politique comme économique), dans les années 80, a refroidi les ardeurs des plus optimistes...Si la progression n'a pas eté vraiment interrompue, elle s'est néanmoins stabilisée. Le tourismeest un secteur plein de promesses mais fragile, vulnérable aux contre-coups de l'histoire. C'estaussi un secteur qui n'a pas atteintsa pleine maturité, traversé de forces et de tendances contradictoires (présence côte à côte de la petite entreprise artisanale et de gros conglomérats, du service humain personnalisé et de la technologie informatique sophistiqué, d'effets positifs et négatifs sur les cultures et l'environne- ment, de courts séjours a proximité de la maison et de longs voyages au bout du monde....). Oui, le tourisme change, mais de manière "énigmatique" et souvent paradoxale.

Mondialisation des marchés, libéralisation des échanges, libre circulation des biens, des personnes, des capitaux, redéfinition des pays, bouleversement des frontières, affirmation des nationalismes, c'est dans ce contexte que le tourisme international évolue "selon des logiques et des dynamiques sociales qui lui servent de cadre " pour reprendre la formulation de Georges Cazes.

Mais alors, quelle formation des ressources humaines? Il faut bien sûr répondre aux besoins de développement des industries du tourisme du Québec; le tourisme québécois est au coeur de la formation a l'Université du Québec à Montréal. Cependant, le système touristique québécois n'est pas fermé, c'est un système ouvert sur le monde en interaction constante avec les autres systèmes nationaux, des pays émetteurs comme récepteurs, des pays du Nord comme du Sud. Ce qui se passe dans ces pays a des incidences surl'évolution du tourismequébécoiset viceversa. La formation doit nécessairement en tenir compte. Mais comment?

\section{Une première réponse: les cours}

Des cours peuvent aborder directement les aspects intemationaux du tourisme: c'est le cas notamment du cours qui a pour titre Dimensions internationales du tourisme dans. le cadre du baccalauréat en gestion et intervention touristiques de l'UQAM; on fait aussi de plus en plus de place aux références venant d'autres pays, aux enjeux internationaux dans les études de cas soumis aux étudiants; des cours de marketing comme des cours de politique ou d'économie ne peuvent plus ignorer les aspects internationaux.

Pour ouvrir davantage leurs horizons, pour mieux saisir la réalité d"un autre environnement, certains étudiants réalisent leur stage de fin d'études dans une entreprise ou une administration nationale $\mathrm{d}^{\dagger}$ un pays étranger. D'autres participent à des "Ecoles intema-
Monsieur Louis Jalinest professeur au Département d'budes urbaines et touristiques de l'UQAM et coordonnateur du CIFORT. 
tionales d'été": il s"agit de cours du programme d'études de l'UQAM en administration, en langues, en politique, en tourisme, etc qui se deroulent exceptionnellement dans un autre pays, done dans un contexte géographique et culturel étranger susceptible d'apporter un nouvel eclairage. Un tel cours, ayant pour titre Installations touristiques, devrait avoir lieu en France à l'été 93; on a recensé, ces dernières années, un certain nombres d'etudiants en tourisme dans des "écoles intemationales d"éte" au Mexique (cours de langue espagnole) ou aux EtatsUnis (gestion, marketing). Uneautre formule connue sous le nom de "projet d' études internationales" est une activité de synthèse de 6 crédits qui vise à faire connaître de façon active un contexte géo-politique étranger et à développer le sens d'entrepreneurship des étudiants participants: la préparation et la recherche de commandites sont à la charge des étudiants dans la session précédant le déplacement à l'étranger. Enfin certains étudiants peuvent suivre une ou deux sessions d'études dans une universite étrangère et les intégrer, s'il y a compatibilité, au cheminement de leur propre programme d'etudes. A l'inverse, l'UQAM accueille dans ses programmes des étudiants venant d'autres universités.

Toutes ces formules - sans compter les voyages éclairs d'observation de quelques jours, non crédités - sont encouragées par l'UQAM; par contre elles sont souvent coûteuses et reposent pour leur réalisation sur l'auto-financement à partir des contributions des étudiants eux-mêmes ou la recherche difficile de commanditaires. L'Université étudie la mise sur pied d'une structure permanente pour appuyer au moins techniquement les étudiants depremier cycle engages dans une démarche internationale.

Tout cela n'est pas suffisant cependant! Au delà des cours, il faut aussi inscrire l'Université dans une réseau international de coopération. C'est le choix qui a tété fait ces dernières années dansle domaine du tourisme. Considérons d'abord le chemin parcouru avant d'exposer certaines perspectives et limites.

\section{Le réseau des centres internationaux de formation et de recherche en tourisme de l'Organisation mondiale du tourisme}

Créce officiellement au milieu des années '70, l'Organisation mondiale du tourisme

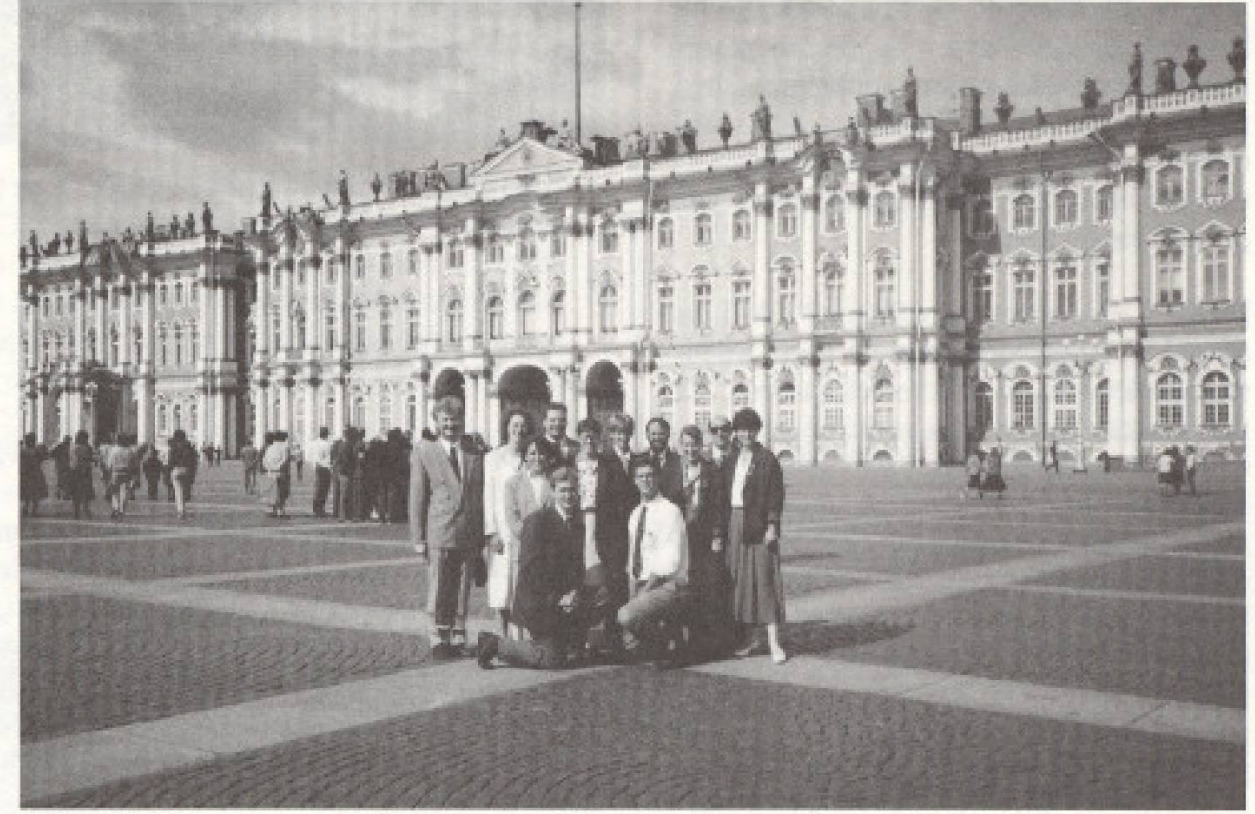

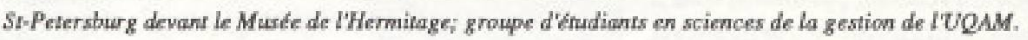

(OMT) regroupe plus de 110 Etats membres et près de 160 membres affiliés du secteur opérationnel. Les questions de formation et d"éducation ont toujours préoccupé cetorganisme international: pendant de nombreuses années, l'OMT avait sa propre école de formation avec la collaboration du gouvemement du pays où elle était installée: les villes de Turin et de Mexico ont donc successivement accueillí cette école qui dispensait des cours de haut niveau à l'intention des candidats soumis par les administrations nationales et les membres affiliés. Des candidats venaient de partout mais particulièrement des pays en voie de développement. Les coûts de fonctionnement d'une telle école étaient élevés et le gouvemement mexicain annonça en 1986 qu'il ne pouvait maintenir son engagement à cet égard.

Que doit faire alors l'OMT? Convaincre un autre gouvernement d'accueillir cette école ou reviser son approche? L'organisation mondiale choisit derevoir son orientation sur cette question et décida de confier la mission à diverses universités ou écoles supérieures réparties géographiquement pour mieux répondre aux besoins des divers pays. En s'appuyant sur un certain nombre d'institutions existantes, 1'OMT reconnaissait par le fait même la qualité de celles-ci.

Informée de cette approche, l'UQAM soumit en 1989 a l'OMT un dossier pour être reconnue comme centre international de formation et de recherche en tourisme. Un premier protocole futsigné entrele recteurde l'UQAM et le secrétaire général de l'OMT à l'automne $89_{*}^{*}$ il fut révisé à l'été 91 et signé à nouveau par le recteur, Claude Corbo, et le secrétaire général, M. Antonio Enriquez Savignace ratifié par l'Assemblée générale de l'OMT a Buenos Aires en septembre 1991.

La reconnaissance de l'UQAM par l'OMT est un honneur qui confirme la qualité de

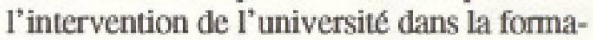
tion touristique de niveau supérieur. Cette reconnaissance entraine certaines obligations morales, notamment l'accueil d'étudiants étrangers en provenance de pays en voie de développement ou la réalisation, dans la mesure du possible, de programmes spécifiques de formation à l'intention de candidats venant des pays membres ou soumis par les membres affiliés. Mais - et c'est peut-être l'intérêt principal - elle inscrit l'UQAM dans un réseau d'une dizaine de centres internationaux qui seront en interrelations les uns, les autres, qui pourront partager leur expertise, dégager des priorités de recherche et d' intervention afin de mieux appréhender les tendances du tourisme international pour les années futures et préciser les orientation que doit prendre la formation des ressources humaine dans un tel contexte.

Outre l'UQAM, le réseau comprend l'Université de Calgary, l'Université George Washington, l'Université d'Hawai, le Centremondial d'études supérieures en tourisme de l'Université de Paris Iet du Conservatoire

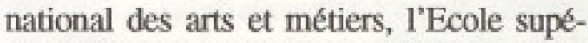
rieure de tourisme de Rome, l'Université de Zagreb, l'Institut supérieur de tourisme de 
Tanger, l'Université de Surrey et l'Ecole hôtelière de La Haye. Le réseau pourra accueillir quelques centres supplémentaires, mais il n'est pas dans l'intention de l'OMT d'en multiplier le nombre afin de maintenir des standards élevés et la qualité des échanges au sein du réseau.

En 1991, les centres accrédités et l'OMT ont adopté un plan stratégique ${ }^{3)}$ pour mieux orienter leurs interventions respectives. Ce plan insistait sur lanécessité de l'information et suggérait la création d'un bulletin périodique sur les questions de formation et de recherche en tourisme, pouvant aussi servir de lien fonctionnel entre les centres. D'emblée dans cetteperspective, Téoros soumitaux membres du réseau le projet de créer Téoros international; cette nouvelle publication, supervisée par un comité scientifique international composé de représentants des diverses institutions du réseau, paraitrait une à deux fois par année, publierait des articles en français, anglais et espagnol, et aurait pour objectif de susciter la réflexion et la mise en commun des connaissances sur la formation supérieure en tourisme, ses tendances, son évolution pour mieux appréhender la complexité du phénomène touristique.

Le projet soumis par l'UQAM fut adopté à Buenos Aires et le travail demise sur pied est en cours.

La participation de l'UQAM au sein du réseau des centres accrédités par l'OMT n'est donc pas passive. L'université entend jouer un rôle actif et en tirer des avantages pour mieux internationaliser son intervention dans les domaines de la formation et de la recherche, seule voie pour répondre aux défis des prochaines années.

\section{Les ententes bilatérales}

Parallèlement à cette approche multilatérale, l'UQAM a conclu des ententes bilatérales de coopération dans le secteur touristique avec des universités françaises: il s'agit de l'Université d'Angers, et plus récemment de l'Université de Toulouse-Le Mirail et du Centre mondial d'études supérieures en tourisme crécé par l'Université de Paris I et du Conservatoire national des Arts et Métiers. Par cesententes, les parties signataires visent à donner un cadre formel à la coopération, à officialiser et à intensifier les échanges déjà amorcés dans les domaines de la formation et de la recherche en tourisme. La cooperation peut prendre diverses formes: réalisation de

TABLE RONDE SUR LA FORMATION EN TOURISME

$\mathrm{XVII}^{\circ}$ assemblée générale de l'Organisation mondiale du Tourisme Buenos Aires, Argentine, 2 octobre 1991

Lors de la demière assemblée générale de l'OMT, une période de temps fut consacrée à la question de la formation en tourisme. Conçue sous la forme d'une table ronde, cette séance de travail a été l'occasion pour certains des participants de faire état des problèmes existant dans ce secteur alors que d'autres en ont profité pour faire une synthèse des programmes mis sur pied dans leur propre établissement pour répondre à la demande croissante d'une formation de qualité et qui réponde aux besoins actuels.

A cette table ronde avaient été invités non seulement des représentants des dix centres de formation reconnus par l'OMT mais aussi d' autres intervenants qui oeuvrent dans le même secteur au plan international. L' animateur de cette session, monsieur Jafar Jafari, éditeur de la revue Annals of Tourism Research (États-Unis), a mis en évidence, en guise d'introduction, l'émergence du tourisme comme sujet de formation académique et comme domaine de recherche. Même si l'intérêt pour la formation en tourisme au niveau universitaire a commencé à poindre dans la première moitié du $\mathrm{XX}^{c}$ siècle, ce n'est vraiment qu'à partir du début des années 1970 que le volet tourisme s'est implanté sérieusement dans plusieurs collèges et universités à travers le monde.

Rappelons également que ce n'est qu'en 1970 que le premier recueil de texteen anglais sur le tourisme a été publiê. C'est tout dire!

Cela dit, les présentations de chacun des participants à la table ronde ont confirmé que les profils en tourisme sont très variables et la coopération à ce niveau au plan national comme au plan international est sérieusement déficiente.

Par ailleurs, le rôle de l'Organisation mondiale du Tourisme par rapport aux dix centres de formation et de recherche, dont l'UQAM, reconnus par cet organisme resteà définir plus étroitement dans le contexte de développement de l'axe Nord-Sud. Et autour de la table, les participants ont émis des points de vue parfois fort différents sur cet aspect fondamental de la relation entre l'OMT et le réseau des centres de formation accrédités. Aussi, ont-ils émis le souhait que la discussion se poursuive à l'intérieur du réseau sur cet aspect crucial pour l'avenir de la formation en tourisme.

\section{Marcel Samson *}

Délégué de l'UQAM à l'assemblée générale de l'OMT

\footnotetext{
- Monsieur Marcel Samson est professeur au Département d'études urbaines et touristiques de I'UQAM et directeur du module de gestion et d'intervention touristiques.
}

projets de recherche conjoints, échanges de professeurset d'experts, réalisation de stages d'études et de perfectionnement pour les étudiants, échanges de documents.

La collaboration entre l'UQAM et certaines universités françaises permet de confronter diverses approches (française, européenne, québécoise, nord-américaine). Malgré des différences de diplômes ou de systèmes éducatifs, une même langue française, a quelques nuances près, et des références culturelles communes militent en faveur d'une telle coopération. D'un point de vue scientique, la collaboration entrel'UQAM et des universités françaises permettra d'ac- croître la quantitéet la qualité des recherches de langue française dans le domaine du tourisme. Cette question de la recherche en langue française est cruciale car il est essentiel d' apporter un point de vue spécifique et distinctif pouréclairer les enjeux du tourisme international et faire un certain contrepoids à l'influence anglosaxonne de plus en plus marquée dans le monde du tourisme et dans celui de la production de l'information et de la recherche touristiques.

De semblables ententes pourraient aussi être conclues dans l'avenir avec d'autres universités ou écoles supérieures en Afrique francophone, en Amérique latine, au Canada 
anglaiset aux Etats-Unis, mais l'insertion de l'UQAMau seindu réseau des centres reconnus par l'OMT permet des échanges avec diverses universités d'Europe, d'Amérique et d'Afrique sans qu'il soitnćcessaire pour le moment de conclure un protocole avec chacune d'elles. Il y a en effet une limite aux ressources que l'ont peut consentir aux échanges internationaux malgré leur interêt manifeste et leur utilité dans la formation en tourisme.

\section{LE CIFORT}

Plusieurs instances au sein de l'UQAM interviennent dans la formation et la recherche en tourisme: module de gestion et intervention touristiques, département d'études urbaines et touristiques, département des sciences administratives, Chaire en tourisme, revue Téoros, Centre de recherche en gestion des services, etc. Sont aussi associés directement à cette intervention le Centre d'études du tourisme, qui bénéficie de l'appui de l'UQAM au titre de partenaire principal, et l'Institut de tourisme et $\mathrm{d}^{*}$ hôttellerie du Québec qui a signé récemment une entente avec l'université pour la réalisation d'une concentration en gestion hôtelière et de restaurants dans le cadre du baccalauréat (licence)en gestionetintervention touristiques.

Dans les rapports avec les interlocuteurs étrangers (OMT, réseau des centres de formation, autres universités), il n'est pas toujoursaiséde préciserles instances del'UQAM concernées dans la formation et la recherche en tourisme; en outre, plusieurs actions impliquent deux ou trois instances de l'université. Aux yeux de l'étranger, la structure matricielle de l'UQAM n'est pas toujours facile à comprendre. C'est donc, a la fois pour une raison de coordination des actions au plan de la coopération internationale et pour offrir une interface unique dans les relations de l'UQAM avec l'étranger dans le domaine du tourisme que fut crés, a la fin de 1991 , le Centre international de formation et de recherche en tourisme (CIFORT). Le Centre est constitué d'un comitế de coordination composé d'un représentant de chaque composante de l'UQAM impliquée dans la formation et la recherche en tourisme et d'un comité consultatif composé de représentants de divers organismes publics et privés du secteur touristique.

En lien avec le Bureau de la coopération internationale de l'UQAM, le CIFORT a pour objectifs, dans les domaine spécifiques de la recherche et de la formation touristiques, de gérer les mandats confiés parl'OMT à l'université comme membre du réseau des centres internationaux de formation et de recherche en tourisme, d'assurer la représentation de l'université auprès de $\mathrm{l}^{4} \mathrm{OMT}$ et lors des diverses réunions du réseau des centres de formation, de coordonner les actions internationales des diverses instances de I'UQAM, de suivre l'évolution des ententes conclues entre l'UQAM et les institutions étrangères, de voiràl'accueil desétudiants et des professeurs étrangers du secteur touristique avec le concours des partenaires concemés.

\section{Un choix calculé}

Tant par les cours dispensés a Montreal ou même, à l'occasion, à l'étranger que par les diverses ententes conclues avec l'Organisation mondiale du tourisme et avec certaines universités, sans oublier cette organisation interne de coordination qu'est le CIFORT, I'UQAM manifeste sa volonté d'inscrire son intervention dans une dynamique internationale. Cette manifestation révèle au grand jour plusieurs convictions: les enjeux du tourisme québécois s'inscrivent dans un contexte de mondialisation des marchés et dans la mouvance du tourisme international, les problèmes du tourisme international sont complexes et nécessitent une approche des questions qui ne se limite pas au seul territoire national, une formation de qualité des étudiants doit être enrichie de l'apport d'expériences de formation vécues dans d'autres pays et de la contribution de recherches effectuces aux quatre coins de la planète, les professeurs ont tout intérêt à confronter avec leurs collègues étrangers leurs methodes pédagogiques et les résultats de leurs recherches pour le bénéfice des étudiants et des milieux professionnels, l'UQAM a une expertise et des compétences qui gagnent àêtre connues a l' étranger, enfin une ouverture sur le monde exige une organisation approprice car la recherche conjointe, les échanges d'étudiants et de professeurs et la réalisation de mandats particuliers de cooperation ne $s^{4}$ improvisent pas.

Dans la formation et la recherche en tourisme, l'UQAMadécidé de s'ouvrir au monde, consciente des défis et des enjeux. Mais ce choix a une limite, celle des ressources humaines et financières quel'on peut consacrer a "l'international", et un risque, celui de la dispersion des efforts. Une fois les ententes conclues, l'infrastructure de base mise en place, il faut livrer la marchandise, ce qui demande d'établir clairement les priorités en termes d'actions et de partenaires. Le CIFORT a justement pour but d'orienter les interventions pour une plus grande efficacité selon les moyens disponibles.

Cettestructure estencore jeune mais prometteuse au plan stratégique.

\section{NOTES ET RÉFÉRENCES}

(1) Georges Cazes, Le tourieme internatlonal - ML rage ou strategle d'avenir?, Paris, Hatier, 1989, p. 191 .

(2) Parmi ceux-ci, Hermann Kahn, cité par Georges Cazes, op. cit. p. 189.

(3) Strategic Mission of WTO Education \& Training Network, texte approuré lors de l'Assemblée générale de l'OMT, septembre 1991. 